\title{
Syntheses of ( \pm )-Haedoxan A, D, E and Their Stereoisomers
}

\author{
Fumito ISHIBASHI and Eiji TANIGUCHI* \\ Department of Agricultural Chemistry, Faculty of Agriculture, \\ Kyushu University, Hakozaki 6-10-1, Higashi-ku, \\ Fukuoka 812, Japan
}

Received December 12, 1988

\begin{abstract}
The naturally occurring insecticides, haedoxans $A$ and $D$, as well as the artificial congener, haedoxan $\mathrm{E}$, and nine of their stereoisomers were synthesized in racemic form.
\end{abstract}

The structure, 1-acetoxy/hydroxy-6-aryl-2aryloxy-3,7-dioxabicyclo[3.3.0]octane, is the representative framework of a series of unique lignans responsible to the insecticidal activity of the plant, Phryma leptostachya L. (Fig. 1). Construction of the framework was accomplished by the syntheses of $( \pm)-\left(1 S^{*}, 2 S^{*}, 5 R^{*}\right.$, $\left.6 S^{*}\right)$-phrymarolin II $(5)^{1)}$ and natural $(+)$ -
$(1 S, 2 S, 5 R, 6 S)$-phrymarolin I (4). ${ }^{2,3)}$ The $2 S$ configuration of 4 has been revised to $2 R$ based on recent NOESY experiments. ${ }^{4}$ Our effort on the Phryma lignans has been extended to the syntheses of the insecticidal neolignans, $(+)$ haedoxan $\mathrm{A}^{5)}$ and $\mathrm{D},{ }^{6)}$ in succession to the preparation of the benzodioxane moiety ${ }^{7}$ involved in these molecules. The strategy de-
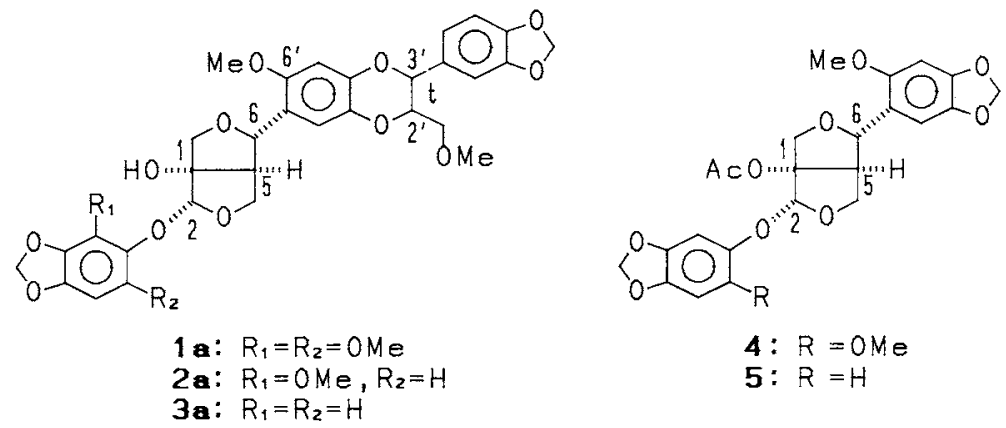

4: $\mathrm{R}=\mathrm{OMe}$

5: $\mathrm{R}=\mathrm{H}$

Fig. 1.
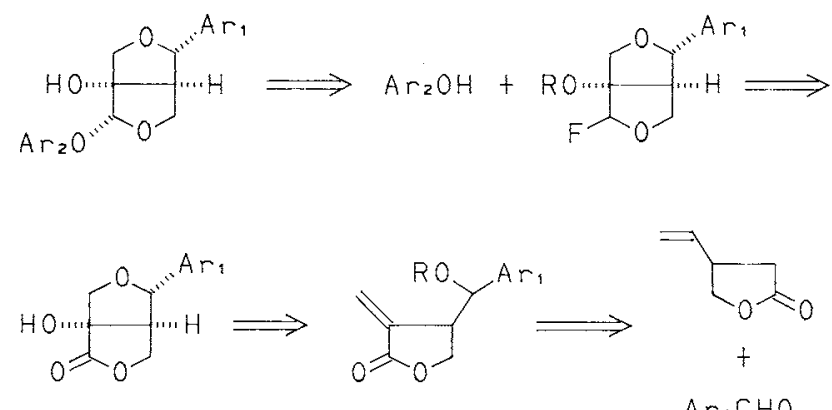

Fig. 2.

* To whom correspondence should be addressed. 

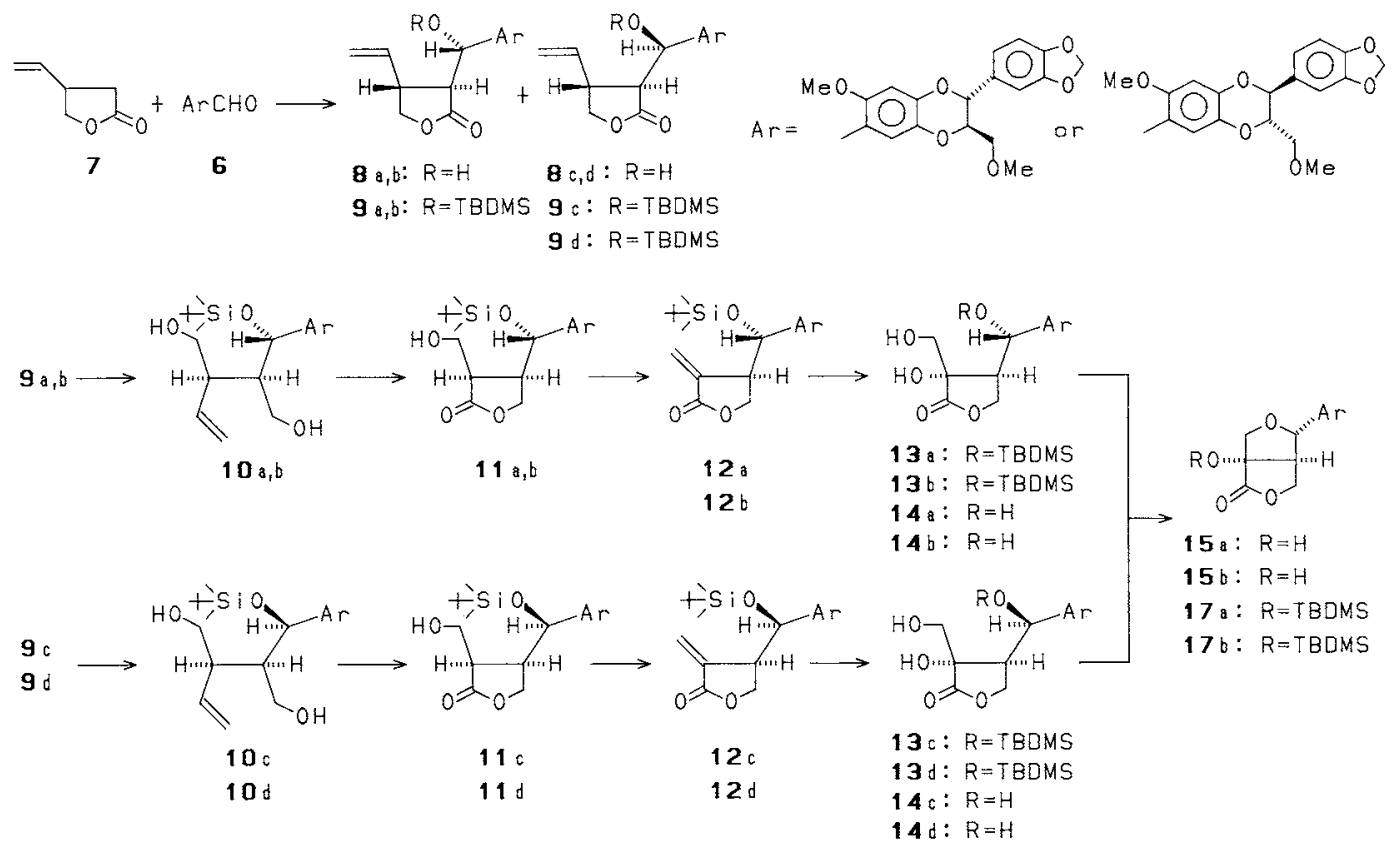

Fig. 3.

veloped for the synthesis of $( \pm)$-phrymarolin II (Fig. 2) was successfully employed for the haedoxan syntheses (Fig. 3). In this paper, the syntheses of ( \pm )-haedoxan A (1a), D (2a) and the congener, haedoxan E (3a), as well as their stereoisomers are described.

The reaction of $( \pm)$-2,3-trans-2,3-dihydro1,4-benzodioxine aldehyde $6^{7)}$ with the lithium enolate of $\beta$-vinyl- $\gamma$-butyrolactone $(7)^{8)}$ generated by lithium diisopropylamide (LDA) in tetrahydrofuran (THF) at $-75^{\circ} \mathrm{C}$ gave a mixture of 2,3-trans-lactonic alcohols $(\mathbf{8 a} \sim \mathbf{d})$ in an appreciable yield. The mixture was almost quantitatively silylated with tert-butyldimethylsilyl triflate (TBDMSOTf) and 2,6-lutidine ${ }^{9)}$ in methylene chloride at $-30^{\circ} \mathrm{C}$ to afford a mixture of four diastereoisomeric tert-butyldimethylsilylated alcohols (TBDMS-ethers, 9a 9d). A conventional method using tertbutyldimethylchlorosilane-imidazole was inefficient for silylation of the benzyl alcohols. The mixture of TBDMS-ethers was separated into three portions, i.e., each of the threo-isomers, $9 \mathrm{c}$ and $9 \mathrm{~d}$, and a mixture of the erythroisomers, $9 \mathbf{a}$ and $\mathbf{9 b}$, in $12 \%, 18 \%$, and $55 \%$ yields, respectively, by means of medium-pres- sure liquid chromatography using silica gel. After desilylating each of the isolates, the stereochemistries of aldols $\mathbf{8 a}, \mathbf{8 b}, \mathbf{8 c}$ and $\mathbf{8 d}$ were assigned on the basis of the coupling constants, $J_{\text {threo }}=6 \sim 9 \mathrm{~Hz}$ and $J_{\text {erythro }}=2 \sim$ $4 \mathrm{~Hz}$, which have been established by House $e t$ al. ${ }^{10)} \mathrm{A}$ lithium aluminum hydride reduction of the mixture of $9 \mathbf{a}$ and $\mathbf{9 b}$ gave a mixture of diols $(\mathbf{1 0 a}$ and $10 \mathrm{~b})$ in a quantitative yield. The inseparable diastereoisomeric diols were together oxidized with a catalytic amount of osmium tetroxide in the presence of a slight excess of $N$-methylmorpholine $N$-oxide ${ }^{11)}$ in a solvent system of tert-BuOH- $\mathrm{Me}_{2} \mathrm{CO}-\mathrm{H}_{2} \mathrm{O}$, and followed by successive oxidation with sodium periodate in aqueous methanol and with silver carbonate-Celite ${ }^{12)}$ in refluxing benzene. A mixture of 2-hydroxymethyllactones (11a and 11b) obtained as a resinous material was treated with mesyl chloride and triethylamine in benzene, and followed by $1,8-$ diazabicyclo[5.4.0]undec-7-ene (DBU) to furnish a mixture of $\alpha$-methylenelactones (12a and $\mathbf{1 2 b}$ ) in $86 \%$ yield. These methylenelactones were separated into each of the diastereoisomers by medium-pressure liquid chro- 
matography using a silica gel column (benzene-EtOAc $=95: 5$ ). A catalytic osmium oxidation of 12a, using $N$-methylmorpholine $N$ oxide as a reoxidant ${ }^{11}$ in a solvent system of tert- $\mathrm{BuOH}-\mathrm{Me}_{2} \mathrm{CO}-\mathrm{THF}-\mathrm{H}_{2} \mathrm{O}$, quantitatively furnished $2 \alpha$-hydroxyl lactone-glycol 13a, which possessed the required configuration for constructing the 3,7-dioxabicyclo[3.3.0]octane framework. The stereoselective oxidation would be controlled by the attack of the oxidant from the less hindered $\alpha$-side of the molecule. After removing the protecting TBDMS group of $13 \mathbf{a}$ with tetra- $n$-butylammonium fluoride ${ }^{13)}$ in THF at room temperature, triol 14a was dehydrated by treating with a catalytic amount of 10-camphorsulfonic acid in methylene chloride at room temperature to furnish the key intermediary $1 \alpha$-hydroxy- $6 \alpha$ $\left[\left(2^{\prime} R^{*}, 3^{\prime} R^{*}\right)-6^{\prime}\right.$-methoxy-2'-methoxymethyl- $3^{\prime}-\left(3^{\prime \prime}, 4^{\prime \prime}-\right.$ methylenedioxy $)$ phenyl-2',3'-dihydro-1',4'-benzodioxin-7'-yl]-3,7-dioxabicyclo[3.3.0]octane-2-one $(\mathbf{1 5 a})^{14)}$ in $84 \%$ yield. The diastereoisomer, $1 \alpha$-hydroxy- $6 \alpha-\left[\left(2^{\prime} S^{*}, 3^{\prime} S^{*}\right)\right.$ -

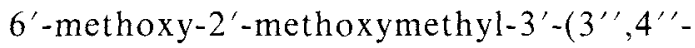
methylenedioxy)phenyl-2',3'-dihydro-1 ', 4'benzodioxin-7'-yl]-3,7-dioxabicyclo[3.3.0]octane-2-one $\mathbf{( 1 5 b )},{ }^{14}$ ) was obtained in $78 \%$ overally yield from $\mathbf{1 2 b}$ by a series of reactions applied to 12a. Desilylation of threo-silyl ether 13d at room temperature furnished triol $\mathbf{1 4 d}$ in a poor yield $(26 \%)$, due to the inapposite product $(16,54 \%)$ resulting from intramolecular transesterification of the benzyloxide anion produced by desilylation of $\mathbf{1 3 d}$. This transesterification of the threo-isomer was overcome by carrying out the desilylation reaction at $0^{\circ} \mathrm{C}$, whereby threo-isomer $13 \mathrm{c}$ afforded $15 \mathbf{a}$ in $63 \%$ yield. The transesterifica-

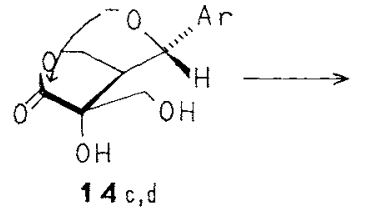

$14 \mathrm{c}, \mathrm{d}$

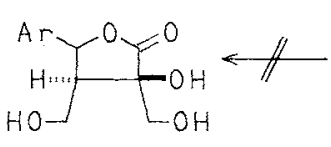

16

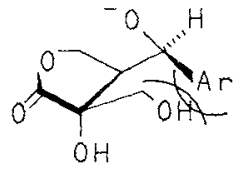

$14 a, b$

Fig. 4.

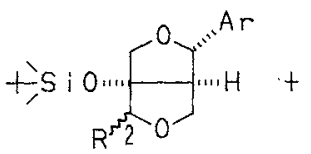

18 a : $R=O H$

$18 b: R=O H$

19 a: $R=F$

$19 b: R=F$

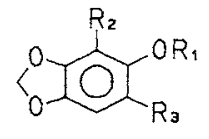

20: $R_{1}=T N S, R_{2}=R_{3}=H$ 21: $R_{1}=I M S, R_{2}=H_{1}, R_{3}=O \mathrm{He}_{\mathrm{e}}$ 22: $R_{1}=H_{1} R_{2}=H_{1} R_{3}=O H_{e}$ 23: $R_{1}=H, R_{2}=R_{3}=$ OMe

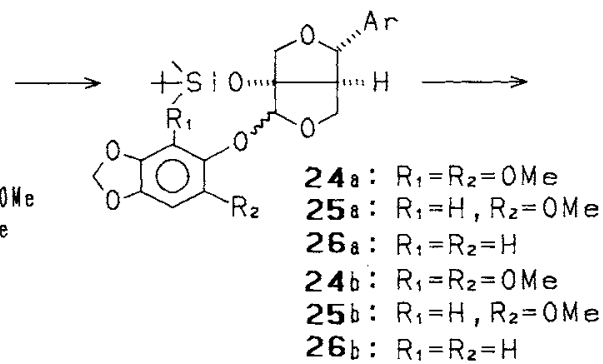

$26 b: R_{1}=R_{2}=H$

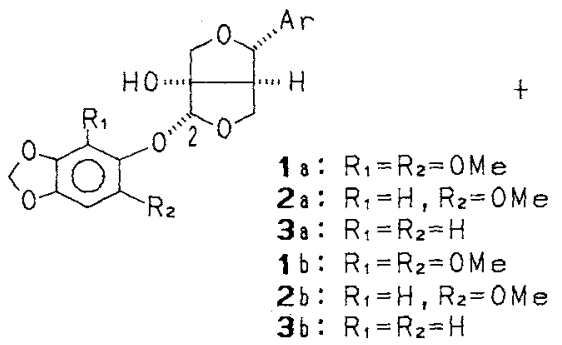

1 a: $R_{1}=R_{2}=O M$ e

$2 \mathrm{a}: \mathrm{R}_{1}=\mathrm{H}, \mathrm{R}_{2}=0 \mathrm{Me}$

$2 b: R_{1}=H, R_{2}=O M e$

$36: R_{1}=R_{2}=H$

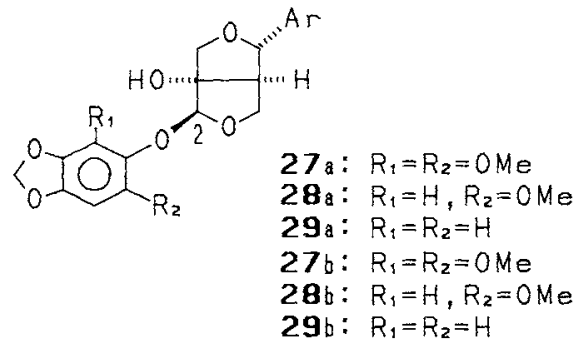

Fig. 5. 
tion would be unfavorable to erythro-isomers 13a and 13b, since steric hindrance between the aromatic group and the hydroxymethyl group in a cis-arrangement on the $\gamma$-butyrolactone ring set the generated benzyloxide anion remote from the lactonic carbonyl group (Fig. 4).

After masking the 1-hydroxyl group of 15a with TBDMSOTf and 2,6-lutidine ${ }^{9}$ in methylene chloride, TBDMS-ether $\mathbf{1 7 a}$ was reduced with diisobutylaluminium hydride (DIBAL) in toluene at $-75^{\circ} \mathrm{C}$ to afford lactol 18a in $94 \%$ yield. This lactol was fluorinated with 2-fluoro-1-methylpyridinium tosylate and triethylamine ${ }^{15)}$ in methylene chloride to give a mixture of the $2 \alpha$ - and $2 \beta$-fluoride (19a) in $81 \%$ yieid (Fig. 5 ). The ratio of $\alpha / \beta$-fluorides was estimated as $1 / 1$ based on the comparable integrated intensities of the resonance signals of $2-\mathrm{H}$ at 5.42 and $5.57 \mathrm{ppm}$, although the respective resonances remained to be assigned to the $\alpha / \beta$-hydrogens. Trimethylsilyl triflate has catalyzed the reactions of $2,3,4,6$-tetra- $O$ benzyl-D-glucopyranosyl fluoride with several trimethylsilylated alcohols. ${ }^{16)}$ The configuration of the anomeric carbon of the glucosides was primarily affected by the solvents used for glycosylation, irrespective of the stereochemistry of the anomeric carbon of the glucosyl fluorides used. The glycosylation reaction in ether resulted in predominant formation of $\alpha$ glucosides from either $\alpha$ - or $\beta$-fluoride, whereas the reaction in acetonitrile favored the formation of $\beta$-glucosides. Treatment of mixed fluorides 19a with $O$-trimethylsilyl (TMS) sesamol (20) in the presence of a catalytic amount of TMSOTf in ether at $0^{\circ} \mathrm{C}$, which was followed by desilylation with tetra- $n$ butylammonium fluoride ${ }^{13)}$ in THF at room temperature, furnished $( \pm$ )-haedoxan $\mathrm{E}(3 \mathbf{a})$ along with $( \pm)$-isohaedoxan $\mathrm{E}(29 \mathrm{a})$, in $25 \%$ and $61 \%$ yields, respectively. The reaction of 19a with $O$-TMS-2-methoxy-4,5-methylenedioxyphenol (21) gave ( \pm )-haedoxan D (2a) and ( \pm )-isohaedoxan D (28a) in $19 \%$ and $72 \%$ yields, respectively. The stereochemical outcome of the $\alpha$-isomers in the acetalization reaction was little improved by applying the method developed for stereoselective preparation of $\alpha$-ribofuranosides from 2,3,5-tri- $O$ benzyl- $\beta$ - $\mathrm{D}$-ribofuranosyl fluoride and various alcohols in the presence of tin(II) chloridetrityl perchlorate in ether. ${ }^{15)}$ Treatment of an equimolar mixture of fluorides 19a with phenol 22 in ether in the presence of tin(II) chloride and trityl perchlorate, which was followed by desilylation of the products, furnished $2 \mathbf{a}$ and $28 \mathbf{a}$ in $25 \%$ and $55 \%$ yields, respectively. Acetalization of 19a with 2,6-dimethoxy-3,4-methylenedioxyphenol (23) in ether in the presence of silver perchlorate and tin(II) chloride, ${ }^{17}$ which was followed by deprotection of the 1-TBDMS group, gave $( \pm)$ haedoxan $A(1 \mathbf{a})$ and ( \pm )-isohaedoxan $A(27 a)$ as well as the $2 \alpha, 6 \beta$-isomer in $17 \%, 16 \%$ and $10 \%$ yields, respectively. Stereoisomers $\mathbf{1 b}, \mathbf{2 b}$, 3b, 27b, 28b and 29b were also synthesized in appreciable yields from the other key intermediary lactone, 15b. The 2,3-dihydro-1,4benzodioxin framework of these stereoisomers should have a relative configuration opposite to that of the dihydrobenzodioxinyl involved in 1a, 2a, 3a, 27a, 28a and 29a.

The ${ }^{1} \mathrm{H}-\mathrm{NMR}$ spectra of synthesized compounds $\mathbf{1 a}$ and $\mathbf{2 a}$, but not of the other stereoisomers, were superimposable on those of natural (+)-haedoxan A and (+)-haedoxan D, respectively. Thus, racemic haedoxan $\mathrm{A}$ and $\mathrm{D}$ were synthesized by 15 steps in $4 \%$ and $3 \%$ yields, respectively. These stereo-controlled syntheses promised the establishment of the absolute configuration of natural haedoxans, employing optically pure $\beta$-vinyl- $\gamma$-butyrolactone $^{3)}$ and 2,3-trans-2,3-dihydro-1,4-benzodioxine aldehyde.

\section{Experimental}

All melting points (mp) are uncorrected. Infrared (IR) spectra were determined on a Shimadzu IR-420 spectrophotometer. ${ }^{1} \mathrm{H}$ - and ${ }^{13} \mathrm{C}-\mathrm{NMR}$ spectra were obtained on a JEOL FX-100 spectrometer. Low-resolution mass spectra (MS) were measured by an ESCO FMD-05A spectrometer at $70 \mathrm{eV}$.

Gravity column chromatography and medium-pressure liquid chromatography were done with Merck silica gel 60 . (70 230 mesh ASTM) and Merck Lichroprep Si60, re- 
spectively. Preparative TLC was performed with Merck silica gel $60 \mathrm{~F}_{254}$ plates $(0.5 \mathrm{~mm}$ thickness, $20 \times 20 \mathrm{~cm})$.

4-Ethenyl-3-[1'-hydroxy-1'-[6'-methoxy-2'-methoxymethyl-3" $-\left(3^{\prime \prime \prime}, 4^{\prime \prime \prime}\right.$-methylenedioxyphenyl $)-2^{\prime \prime}, 3^{\prime \prime}-d i$ hydro-1" ',4"-benzodioxin-7"'-yl]methyl]dihydro-2(3H)furanones $(\mathbf{8} \mathbf{a} \sim \mathbf{8 d})$. LDA was prepared by adding a $15 \%$ solution of $n$-BuLi in $n$-hexane $(4.20 \mathrm{ml}, 6.74 \mathrm{mmol})$ to a solution of diisopropylamine $(0.94 \mathrm{ml}, 6.74 \mathrm{mmol})$ in dry THF $(50 \mathrm{ml})$ at $-30^{\circ} \mathrm{C}$ under argon and stirring for $15 \mathrm{~min}$ at $-10^{\circ} \mathrm{C}$. A solution of lactone $7(0.76 \mathrm{~g}, 6.79 \mathrm{mmol})$ in THF $(15 \mathrm{ml})$ was added in drops to the LDA solution at $-75^{\circ} \mathrm{C}$. After $30 \mathrm{~min}$, aldehyde $6(2.01 \mathrm{~g}, 5.61 \mathrm{mmol})$ dissolved in THF ( $15 \mathrm{ml}$ ) was also added in drops. After $3 \mathrm{hr}$, the reaction was quenched with $10 \%$ aqueous $\mathrm{NH}_{4} \mathrm{Cl}$. The mixture was warmed to room temperature and extracted with ether. The aqueous layer was re-extracted with AcOEt, and the combined extracts were washed with $5 \%$ aquoeus $\mathrm{NaHCO}_{3}$, dried over $\mathrm{Na}_{2} \mathrm{SO}_{4}$, and evaporated. The oily residue was chromatographed on silica gel eluted with benzene-EtOAc $(4: 1)$ to give a mixture of $\mathbf{8 a} \sim \mathbf{8 d}(2.64 \mathrm{~g}, 5.61 \mathrm{mmol})$ as a viscous oil in a quantitative yield. IR $v_{\max }\left(\mathrm{CHCl}_{3}\right) \mathrm{cm}^{-1}: 3100 \sim 3650(\mathrm{OH})$, $1760(\mathrm{C}=\mathrm{O}), 1601,1500,1445,1160,1065,1020$. MS $m / z: 470\left(\mathrm{M}^{+}\right), 359,358,327,326,192,161,160$ (base), 131, 103, 54. Anal. Found: C, 63.77; H, 5.56. Calcd. for $\mathrm{C}_{25} \mathrm{H}_{26} \mathrm{O}_{9}: \mathrm{C}, 63.83 ; \mathrm{H}, 5.53 \%$.

3-[ $I^{\prime}$-[(tert-Butyldimethylsilyl)oxy]- $I^{\prime}-\left[\sigma^{\prime \prime}-\right.$ methoxy$2^{\prime \prime}$-methoxymethyl-3" $-\left(3^{\prime \prime}, 44^{\prime \prime}\right.$-methylenedioxyphenyl)-2" ,3"'-dihydro-1" ,4" -benzodioxin-7"'-yl]methyl]4-ethenyldihydro-2(3H)-furanones $(9 \mathbf{a} \sim 9 \mathrm{~d})$. To a stirred solution of $\mathbf{8 a} \sim \mathbf{8 d}(2.64 \mathrm{~g}, 5.61 \mathrm{mmol})$ and 2,6 -lutidine (1.31 ml, $11.25 \mathrm{mmol}$ ) in dry $\mathrm{CH}_{2} \mathrm{Cl}_{2}$ was added in drops tert $-\mathrm{BuMe}_{2} \mathrm{SiOTf}(1.93 \mathrm{ml}, 8.42 \mathrm{mmol})$ in a solution of $\mathrm{CH}_{2} \mathrm{Cl}_{2}(10 \mathrm{ml})$ at $-40^{\circ} \mathrm{C}$ under argon. After $35 \mathrm{~min}$ at $-30^{\circ} \mathrm{C}$, the reaction was quenched with $5 \%$ aqueous $\mathrm{NaHCO}_{3}$. The $\mathrm{CH}_{2} \mathrm{Cl}_{2}$ layer was separated and the aqueous layer was extracted with $\mathrm{CH}_{2} \mathrm{Cl}_{2}$. The combined extracts were dried over $\mathrm{Na}_{2} \mathrm{SO}_{4}$ and evaporated. The oily residue was purified by medium-pressure liquid chromatography on silica gel. Elution with $5 \%$ EtOAc in benzene first afforded a mixture of $9 \mathrm{a}$ and $9 \mathrm{~b}(1.79 \mathrm{~g}, 3.07 \mathrm{mmol}$, $55 \%$ yield), was followed by $9 \mathrm{c}(0.41 \mathrm{~g}, 0.70 \mathrm{mmol}, 12 \%$ yield), and finally $9 \mathrm{~d}(0.58 \mathrm{~g}, 0.99 \mathrm{mmol}, 18 \%$ yield $)$. The mixture of $9 \mathrm{a}$ and $9 \mathrm{~b}: \mathrm{mp} 141 \sim 150^{\circ} \mathrm{C}$. 9c. $\mathrm{mp} 14 \mathrm{I} \sim 144^{\circ} \mathrm{C}$. NMR $\delta_{\mathrm{H}}\left(\mathrm{CDCl}_{3}\right):-0.05(3 \mathrm{H}, \mathrm{s}), 0.12(3 \mathrm{H}, \mathrm{s}), 0.94(9 \mathrm{H}$, s), $2.71(1 \mathrm{H}, \mathrm{dd}, J=4,8 \mathrm{~Hz}), 3.05(1 \mathrm{H}, \mathrm{m}), 3.27(1 \mathrm{H}, \mathrm{dd}$, $J=4,10 \mathrm{~Hz}), 3.33(3 \mathrm{H}, \mathrm{s}), 3.59(1 \mathrm{H}, \mathrm{dd}, J=3,10 \mathrm{~Hz}), 3.68$ $(3 \mathrm{H}, \mathrm{s}), 3.81(1 \mathrm{H}, \mathrm{dd}, J=7,9 \mathrm{~Hz}), 3.98(1 \mathrm{H}, \mathrm{m}), 4.14(1 \mathrm{H}$, $\mathrm{dd}, J=8,9 \mathrm{~Hz}), 4.88 \sim 5.33(4 \mathrm{H}, \mathrm{m}), 5.52 \sim 5.88(1 \mathrm{H}, \mathrm{m})$, $5.97(2 \mathrm{H}, \mathrm{s}), 6.4 \mathrm{l}(\mathrm{lH}, \mathrm{s}), 6.72 \sim 6.96(3 \mathrm{H}, \mathrm{m}), 7.18(\mathrm{lH}, \mathrm{s})$. 9d. $\mathrm{mp} 142 \sim 145^{\circ} \mathrm{C}$. NMR $\delta_{\mathrm{H}}\left(\mathrm{CDCl}_{3}\right):-0.06(3 \mathrm{H}, \mathrm{s})$, $0.12(3 \mathrm{H}, \mathrm{s}), 0.93(9 \mathrm{H}, \mathrm{s}), 2.70(1 \mathrm{H}, \mathrm{dd}, J=4,8 \mathrm{~Hz}), 3.00$ $(1 \mathrm{H}$, deformed $\mathrm{t}, J=7 \mathrm{~Hz}), 3.26(1 \mathrm{H}, \mathrm{dd}, J=4,11 \mathrm{~Hz})$, $3.32(3 \mathrm{H}, \mathrm{s}), 3.63(1 \mathrm{H}, \mathrm{dd}, J=3,11 \mathrm{~Hz}), 3.67(3 \mathrm{H}, \mathrm{s})$, $3.68 \sim 4.14(3 \mathrm{H}, \mathrm{m}), 4.84 \sim 5.38(4 \mathrm{H}, \mathrm{m}), 5.50 \sim 5.92(1 \mathrm{H}$, $\mathrm{m}), 5.96(2 \mathrm{H}, \mathrm{s}), 6.41(1 \mathrm{H}, \mathrm{s}), 6.72 \sim 6.96(3 \mathrm{H}, \mathrm{m}), 7.19$ (1 H. br.s). IR $v_{\max }\left(\mathrm{CHCl}_{3}\right) \mathrm{cm}^{-1}: 3350,1760,1601,1500$, 1445, 1160, 1065, 1021. Anal. Found: C, 63.71; H, 6.92. Calcd. for $\mathrm{C}_{31} \mathrm{H}_{40} \mathrm{O}_{9}$ Si: C, $63.70 ; \mathrm{H}, 6.85 \%$

2-[1'-[(tert-Butyldimethylsilyl)oxy]- $l^{\prime}-\left[6^{\prime \prime}-\right.$ methoxy$2^{\prime \prime}$-methoxymethyl-3"'-(3"',4"' -methylenedioxyphenyl $)$ $2 ", 33^{\prime \prime}$-dihydro-1" 4 "'-benzodioxin-7"'-yl] methyl]-3ethenyl-1,4-butanediols (10a and $\mathbf{1 0 b}$ ). To a stirred slurry of $\mathrm{LiAlH}_{4}(0.14 \mathrm{~g}, 3.68 \mathrm{mmol})$ in dry ether $(30 \mathrm{ml})$ was added in drops a solution of a mixture of $9 \mathrm{a}$ and $9 \mathrm{~b}(2.02 \mathrm{~g}$, $3.46 \mathrm{mmol})$ in dry THF $(30 \mathrm{ml})$ at $-10^{\circ} \mathrm{C}$ under argon. After $30 \mathrm{~min}$ at $-10^{\circ} \mathrm{C}$, the excess reagent was decomposed by carefully adding EtOAc, and then $10 \%$ aqueous citric acid. The organic layer was separated and the aqueous layer was extracted with EtOAc. The combined organic extracts were washed with $5 \%$ aqueous $\mathrm{NaHCO}_{3}$, dried over $\mathrm{Na}_{2} \mathrm{SO}_{4}$, and evaporated. The oily residue was chromatographed on silica gel eluted with $n$-hexaneEtOAc $(1: 1)$ to give a mixture of $10 \mathrm{a}$ and $10 \mathrm{~b}(2.00 \mathrm{~g}$, $3.40 \mathrm{mmol})$ as a viscous oil in $98 \%$ yield. IR $v_{\max }\left(\mathrm{CHCl}_{3}\right)$ $\mathrm{cm}^{-1}: 3100 \sim 3650(\mathrm{OH}), 2950,1602,1502,1445,1255(\mathrm{C}-$ Si), 1197, 1155, 1068, 1043. Anal. Found: C, 62.81; H, 7.53. Calcd. for $\mathrm{C}_{31} \mathrm{H}_{44} \mathrm{O}_{9} \mathrm{Si}: \mathrm{C}, 63.24 ; \mathrm{H}, 7.53 \%$.

4-[1'-[(tert-Butyldimethylsilyl)oxy] $-1^{\prime}-\left[6^{\prime \prime}-\right.$ methoxy-2"'-

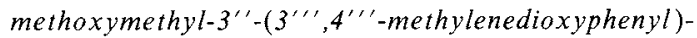
$2^{\prime \prime}, 3^{\prime \prime}$-dihydro-1", 4"'-benzodioxin-7"'-yl]methyl $]-3-h y d r o-$ xymethyldihydro-2(3H)-furanones (11a and 11b). A solution of a mixture of $10 \mathrm{a}$ and $10 \mathrm{~b}(2.00 \mathrm{~g}, 3.40 \mathrm{mmol}), N$ methylmorpholine $N$-oxide monohydrate $(0.52 \mathrm{~g}, 3.85$ mmol) and $2 \%$ aqueous $\mathrm{OsO}_{4}(1 \mathrm{ml})$ in a mixture of acetone $(20 \mathrm{ml})$, tert $-\mathrm{BuOH}(5 \mathrm{ml})$ and water $(5 \mathrm{ml})$ was stirred at room temperature for $18 \mathrm{hr}$ under argon. Celite and aqueous $\mathrm{NaHSO}_{3}(1 \mathrm{~g} / 20 \mathrm{ml})$ were added, and the resulting mixture was filtered. The residue was washed well with acetone and the filtrate was concentrated under reduced pressure. The oily residue was taken up in EtOAc, washed with brine, dried over $\mathrm{Na}_{2} \mathrm{SO}_{4}$, and evaporated to give an oil $(2.45 \mathrm{~g})$.

The crude product was dissolved in $\mathrm{MeOH}(40 \mathrm{ml})$, and aqueous $\mathrm{NaIO}_{4}(0.37 \mathrm{~g}, 1.73 \mathrm{mmol} / 10 \mathrm{ml})$ was added in drops at $0^{\circ} \mathrm{C}$. After $20 \mathrm{~min}$ at $0^{\circ} \mathrm{C}$, the reaction was worked up with $10 \%$ aqueous $\mathrm{Na}_{2} \mathrm{SO}_{3}(10 \mathrm{ml})$. The methanol was removed under reduced pressure, and the residue was extracted twice with EtOAc. The extracts were washed with brine, dried over $\mathrm{Na}_{2} \mathrm{SO}_{4}$, and evaporated to give an oil $(2.05 \mathrm{~g})$.

A mixture of the crude lactols and $\mathrm{Ag}_{2} \mathrm{CO}_{3} /$ Celite (5.1 g, containing c. $5.1 \mathrm{mmol}$ of $\left.\mathrm{Ag}_{2} \mathrm{CO}_{3}\right)$ in benzene $(50 \mathrm{ml})$ was heated under reflux for $30 \mathrm{~min}$. The mixture was then filtered and the residue was washed well with benzene. The filtrate was concentrated to give a mixture of $11 \mathbf{a}$ and $\mathbf{1 1} \mathbf{b}$ $(1.96 \mathrm{~g})$ as a viscous oil, which was used for the next step without further purifications. An analytically pure sample was obtained by preparative TLC on silica gel. IR $v_{\text {max }}\left(\mathrm{CHCl}_{3}\right) \mathrm{cm}^{-1}: 835,1067,1250(\mathrm{C}-\mathrm{Si}), 1310,1500$, 
1602, $1702(\mathrm{C}=\mathrm{O}), 3100 \sim 3600(\mathrm{OH})$. Anal. Calcd. for $\mathrm{C}_{30} \mathrm{H}_{40} \mathrm{O}_{10} \mathrm{Si}: \mathrm{C}, 61.21 ; \mathrm{H}, 6.85$. Found: $\mathrm{C}, 61.04 ; \mathrm{H}$, $6.78 \%$

4-[1'-[(tert-Butyldimethylsilyl)oxy]-1'-[6"-methoxy$2^{\prime \prime}$-methoxymethyl-3" $-\left(3^{\prime \prime}, 4^{\prime \prime}\right.$-methylenedioxyphenyl $)$ $2^{\prime \prime}, 3^{\prime \prime}$-dihydro-1", 4" -benzodioxin-7" -yl ] methyl]-3methylenedihydro-2(3H)-furanones $(12 \mathbf{a} \sim 12 \mathrm{~d})$. Methanesulfonyl chloride $(0.39 \mathrm{ml}, 5.10 \mathrm{mmol})$ was added to a stirred solution of a mixture of $11 \mathrm{a}$ and $11 \mathrm{~b}(1.96 \mathrm{~g})$ and triethylamine $(0.95 \mathrm{ml}, 6.80 \mathrm{mmol})$ in dry benzene $(50 \mathrm{ml})$ at $0^{\circ} \mathrm{C}$. After $2.5 \mathrm{hr}$ at room temperature, diazabicyclo[5.4.0]undec-7-ene $(0.78 \mathrm{~g}, 5.10 \mathrm{mmol})$ was added, and stirring was continued for $45 \mathrm{~min}$. The mixture was then diluted with EtOAc, washed successively with $10 \%$ aqueous citric acid and $5 \%$ aqueous $\mathrm{NaHCO}_{3}$, dried over $\mathrm{Na}_{2} \mathrm{SO}_{4}$, and evaporated. The oily residue was chromatographed on silica gel with $5 \%$ EtOAc in benzene as the eluant to give a mixture of $12 \mathrm{a}$ and $12 \mathbf{b}(1.67 \mathrm{~g}, 2.93 \mathrm{mmol})$ in $86 \%$ overall yield from diols $10 \mathrm{a}$ and $10 \mathrm{~b}$. The mixture was separated by medium-pressure liquid chromatography on silica gel eluted with the same solvent to first afford $\mathbf{1 2 b}$ $(0.53 \mathrm{~g}$, Rf $0.49,10 \%$ EtOAc in benzene), then a mixture of $12 \mathrm{a}$ and $12 \mathrm{~b}(0.18 \mathrm{~g})$, and finally $12 \mathrm{a}(0.96 \mathrm{~g}, R f 0.44,10 \%$ EtOAc in benzene).

12a. A viscous oil. NMR $\delta_{\mathrm{H}}\left(\mathrm{CDCl}_{3}\right):-0.19(3 \mathrm{H}, \mathrm{s})$, $0.04(3 \mathrm{H}, \mathrm{s}), 0.87(9 \mathrm{H}, \mathrm{s}), 3.08 \sim 3.40(2 \mathrm{H}, \mathrm{m}), 3.33(3 \mathrm{H}, \mathrm{s})$, $3.62(1 \mathrm{H}, \mathrm{dd}, J=3,12 \mathrm{~Hz}), 3.71(3 \mathrm{H}, \mathrm{s}), 3.96(1 \mathrm{H}, \mathrm{m})$, $4.07 \sim 4.38(2 \mathrm{H}, \mathrm{m}), 4.99(\mathrm{IH}, \mathrm{d}, J=8 \mathrm{~Hz}), 5.05(1 \mathrm{H}, \mathrm{d}, J=$ $6 \mathrm{~Hz}), 5.33(1 \mathrm{H}$, br. s), $5.97(2 \mathrm{H}, \mathrm{s}), 6.22(1 \mathrm{H}$, br.s $), 6.45$ $(1 \mathrm{H}, \mathrm{s}), 6.74 \sim 6.90(3 \mathrm{H}, \mathrm{m}), 6.96(1 \mathrm{H}, \mathrm{s}) . \mathrm{IR} v_{\max }\left(\mathrm{CHCl}_{3}\right)$ $\mathrm{cm}^{-1}: 2950,1755(\mathrm{C}=\mathrm{O}), 1600,1504,1493,1465,1448$, 1309,1253 (C-Si), 1195, 1163, 1121, 1095, 1067, 1039, 857, 837.

12b. A viscous oil. NMR $\delta_{\mathrm{H}}\left(\mathrm{CDCl}_{3}\right):-0.19(3 \mathrm{H}, \mathrm{s})$, $0.03(3 \mathrm{H}, \mathrm{s}), 0.87(9 \mathrm{H}, \mathrm{s}), 3.12 \sim 3.42(3 \mathrm{H}, \mathrm{m}), 3.33(3 \mathrm{H}, \mathrm{s})$, $3.54(1 \mathrm{H}, \mathrm{dd}, J=3,12 \mathrm{~Hz}), 3.71(3 \mathrm{H}, \mathrm{s}), 3.98(1 \mathrm{H}, \mathrm{m})$, $4.09 \sim 4.26(2 \mathrm{H}, \mathrm{m}), 4.95(1 \mathrm{H}, \mathrm{d}, J=8 \mathrm{~Hz}), 5.04(1 \mathrm{H}, \mathrm{d}, J=$ $7 \mathrm{~Hz}$ ), 5.34 (1H, br.s), $5.97(2 \mathrm{H}, \mathrm{s}), 6.23$ (1H, br.s), 6.45 $(1 \mathrm{H}, \mathrm{s}), 6.74 \sim 6.89(3 \mathrm{H}, \mathrm{m}), 6.95(1 \mathrm{H}, \mathrm{s})$. Anal. Calcd. for $\mathrm{C}_{30} \mathrm{H}_{38} \mathrm{O}_{9} \mathrm{Si}: \mathrm{C}, 63.14 ; \mathrm{H}, 6.71$. Found: $\mathrm{C}, 62.82 ; \mathrm{H}, 6.70 \%$.

12c. A viscous oil. NMR $\delta_{\mathrm{H}}\left(\mathrm{CDCl}_{3}\right):-0.16(3 \mathrm{H}, \mathrm{s})$, $0.00(3 \mathrm{H}, \mathrm{s}), 0.90(9 \mathrm{H}, \mathrm{s}), 3.16 \sim 3.41(2 \mathrm{H}, \mathrm{m}), 3.34(3 \mathrm{H}$, s), $3.55(1 \mathrm{H}, \mathrm{dd}, J=3,11 \mathrm{~Hz}), 3.72(3 \mathrm{H}, \mathrm{s}), 3.97(1 \mathrm{H}, \mathrm{m})$, $4.13(1 \mathrm{H}, \mathrm{dd}, J=8,9 \mathrm{~Hz}), 4.49(1 \mathrm{H}, \mathrm{dd}, J=4,9 \mathrm{~Hz}), 4.97$ $(1 \mathrm{H}, \mathrm{d}, J=8 \mathrm{~Hz}), 5.12(1 \mathrm{H}, \mathrm{d}, J=5 \mathrm{~Hz}), 5.42(1 \mathrm{H}, \mathrm{d}, J=$ $2 \mathrm{~Hz}), 5.98(2 \mathrm{H}, \mathrm{s}), 6.24(1 \mathrm{H}, \mathrm{d}, J=2 \mathrm{~Hz}), 6.44(1 \mathrm{H}, \mathrm{s})$, $6.72 \sim 6.94(3 \mathrm{H}, \mathrm{m}), 7.04(1 \mathrm{H}, \mathrm{s})$.

12d. mp $120 \sim 124^{\circ} \mathrm{C}$. NMR $\delta_{\mathrm{H}}\left(\mathrm{CDCl}_{3}\right):-0.16(3 \mathrm{H}, \mathrm{s})$, $0.00(3 \mathrm{H}, \mathrm{s}), 0.89(9 \mathrm{H}, \mathrm{s}), 3.12 \sim 3.44(2 \mathrm{H}, \mathrm{m}), 3.33(3 \mathrm{H}, \mathrm{s})$, $3.66(1 \mathrm{H}, \mathrm{dd}, J=3,11 \mathrm{~Hz}), 3.72(3 \mathrm{H}, \mathrm{s}), 3.88 \sim 4.24(2 \mathrm{H}$, $\mathrm{m}), 4.47(1 \mathrm{H}, \mathrm{dd}, J=5,9 \mathrm{~Hz}), 5.00(1 \mathrm{H}, \mathrm{d}, J=9 \mathrm{~Hz}), 5.11$ $(1 \mathrm{H}, \mathrm{d}, J=5 \mathrm{~Hz}), 5.40(1 \mathrm{H}, \mathrm{d}, J=2 \mathrm{~Hz}), 5.99(2 \mathrm{H}, \mathrm{s}), 6.23$ $(\mathrm{lH}, \mathrm{d}, J=2 \mathrm{~Hz}), 6.44(1 \mathrm{H}, \mathrm{s}), 6.72 \sim 6.98(3 \mathrm{H}, \mathrm{m}), 7.06$ (lH, s).

4-[I'-[(tert-Butyldimethylsilyl)oxy $]-I^{\prime}-\left\{6^{\prime \prime}-\right.$ methoxy-

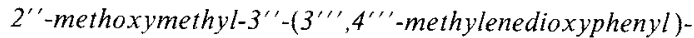
2" ,3"'-dihydro-1" ,4" -benzodioxin-7''-yl]methyl]-3hydroxy-3-(hydroxymethyl)dihydro-2(3H)-furanone

(13a). A solution of $12 \mathrm{a}(0.13 \mathrm{~g}, 0.23 \mathrm{mmol}), N$-methylmorpholine $N$-oxide monohydrate $(0.04 \mathrm{~g}, 0.30 \mathrm{mmol})$ and $2 \%$ aqueous $\mathrm{OsO}_{4}(0.2 \mathrm{ml})$ in a mixture of acetone $(6 \mathrm{ml})$, THF $(0.5 \mathrm{ml})$, tert-BuOH $(2 \mathrm{ml})$ and water $(2 \mathrm{ml})$ was stirred at room temperature in the dark for $14 \mathrm{hr}$ under argon. Celite and aqueous $\mathrm{NaHSO}_{3}(0.2 \mathrm{~g} / 10 \mathrm{ml})$ were added, and the mixture was filtered. The residue was washed well with acetone, and the filtrate was concentrated under reduced pressure. The residue was taken up in EtOAc, washed with brine, dried over $\mathrm{Na}_{2} \mathrm{SO}_{4}$, and concentrated. The oily residue was chromatographed on silica gel eluted with $n$ hexane-EtOAc $(1: 2)$ to give $13 \mathfrak{a}(0.13 \mathrm{~g}, 0.22 \mathrm{mmol})$ as a viscous oil in $96 \%$ yield. NMR $\delta_{\mathrm{H}}\left(\mathrm{CDCl}_{3}\right):-0.26(3 \mathrm{H}, \mathrm{s})$, $0.10(3 \mathrm{H}, \mathrm{s}), 0.91(9 \mathrm{H}, \mathrm{s}), 2.52 \sim 2.76(1 \mathrm{H}, \mathrm{br}), 2.92 \sim 3.16$ $(2 \mathrm{H}, \mathrm{m}), 3.27(1 \mathrm{H}, \mathrm{dd}, J=3,11 \mathrm{~Hz}), 3.33(3 \mathrm{H}, \mathrm{s}), 3.63(1 \mathrm{H}$, dd, $J=3,11 \mathrm{~Hz}), 3.73(3 \mathrm{H}, \mathrm{s}), 3.84 \sim 4.24(5 \mathrm{H}, \mathrm{m}), 5.00$ $(1 \mathrm{H}, \mathrm{d}, J=8 \mathrm{~Hz}), 5.28(1 \mathrm{H}, \mathrm{d}, J=8 \mathrm{~Hz}), 5.98(2 \mathrm{H}, \mathrm{s}), 6.47$ $(1 \mathrm{H}, \mathrm{s}), 6.80 \sim 6.96(3 \mathrm{H}, \mathrm{m}), 7.00(1 \mathrm{H}, \mathrm{s}) . \mathrm{IR} v_{\max }\left(\mathrm{CHCl}_{3}\right)$ $\mathrm{cm}^{-1}: 838,1018(\mathrm{C}-\mathrm{O}), 1040(\mathrm{C}-\mathrm{O}), 1250(\mathrm{C}-\mathrm{Si}), 1315$, 1495, 1602, $1777(\mathrm{C}=\mathrm{O}), 3200 \sim 3600(\mathrm{OH})$. Anal. Calcd. for $\mathrm{C}_{30} \mathrm{H}_{40} \mathrm{O}_{1 \mathrm{~L}} \mathrm{Si:} \mathrm{C}, 59.59 ; \mathrm{H}, 6.67$. Found: $\mathrm{C}, 59.42 ; \mathrm{H}$, $6.71 \%$.

1-Hydroxy-6-[6'-methoxy-2'-methoxymethyl- $3^{\prime}-\left(3^{\prime \prime}, 4^{\prime \prime}\right.$ methylenedioxyphenyl)-2',3'-dihydro- $I^{\prime}, 4^{\prime}$ - benzodioxin- ${ }^{\prime}$ '$y l]$-3,7-dioxabicyclo[3.3.0]octan-2-ones (15a and 15b). A $1 \mathrm{M}$ solution of $n-\mathrm{Bu}_{4} \mathrm{NF}$ in THF $(0.27 \mathrm{ml}, 0.27 \mathrm{mmol})$ was added to a solution of $13 \mathrm{a}(0.13 \mathrm{~g}, 0.22 \mathrm{mmol})$ in dry THF $(5 \mathrm{ml})$ at $0^{\circ} \mathrm{C}$, and the mixture was stirred for $1.5 \mathrm{hr}$ at $0^{\circ} \mathrm{C}$. The reaction was quenched by $10 \%$ aqueous $\mathrm{NH}_{4} \mathrm{Cl}$, and the mixture was then extracted twice with EtOAc. The extracts were washed with brine, dried over $\mathrm{Na}_{2} \mathrm{SO}_{4}$, and evaporated. The oily residue was chromatographed on silica gel eluted with EtOAc-THF $(3: 1)$ to give triol $14 a$ as a viscous oil $(0.67 \mathrm{~g})$.

The triol was dissolved in dry $\mathrm{CH}_{2} \mathrm{Cl}_{2}(10 \mathrm{ml})$, and a catalytic amount of CSA (c. $20 \mathrm{mg}$ ) was added. After $16 \mathrm{hr}$ at room temperature, the reaction was quenched by adding one drop of triethylamine. The solvent was removed under reduced pressure, and the oily residue was chromatographed on silica gel with $n$-hexane-EtOAc $(1: 2)$ as the eluant to give lactone $15 \mathrm{a}(0.09 \mathrm{~g}, 0.19 \mathrm{mmol})$ as white crystals in $87 \%$ yield, mp $177 \sim 179^{\circ} \mathrm{C}$. NMR $\delta_{\mathrm{H}}$ $\left(\mathrm{CDCl}_{3}\right): 2.98(1 \mathrm{H}, \mathrm{s}), 3.12(1 \mathrm{H}, \mathrm{m}), 3.27(\mathrm{lH}, \mathrm{dd}, J=5$, $11 \mathrm{~Hz}), 3.33(3 \mathrm{H}, \mathrm{s}), 3.58(1 \mathrm{H}, \mathrm{dd}, J=3,11 \mathrm{~Hz}), 3.73(3 \mathrm{H}$, s), $3.97(1 \mathrm{H}, \mathrm{m}), 4.18(2 \mathrm{H}, \mathrm{s}), 4.34(1 \mathrm{H}, \mathrm{dd}, J=6,11 \mathrm{~Hz})$, $4.65(1 \mathrm{H}, \mathrm{dd}, J=9,10 \mathrm{~Hz}), 4.96(1 \mathrm{H}, \mathrm{d}, J=8 \mathrm{~Hz}), 5.10$ $(1 \mathrm{H}, \mathrm{d}, J=5 \mathrm{~Hz}), 5.97(2 \mathrm{H}, \mathrm{s}), 6.49(1 \mathrm{H}, \mathrm{s}), 6.72 \sim 6.92$ $(3 \mathrm{H}, \mathrm{m}), 7.14(1 \mathrm{H}, \mathrm{s}) . \mathrm{IR} v_{\max }\left(\mathrm{CHCl}_{3}\right) \mathrm{cm}^{-1}: 3100 \sim 3650$ $(\mathrm{OH}), 1777(\mathrm{C}=\mathrm{O}), 1601,1502,1495,1469,1450,1335$, $1255,1200,1170,1125,1073,1042,1205$. MS $m / z: 472$ $\left(\mathrm{M}^{+}\right), 192$ (base), 162, 161, 160, 135, 131, 103. Anal. Found: C, 60.99; H, 5.17. Calcd. for $\mathrm{C}_{24} \mathrm{H}_{24} \mathrm{O}_{10}: \mathrm{C}, 61.02$; $\mathrm{H}, 5.08 \%$. 
In the same manner as that just described, lactone $\mathbf{1 5 b}$ was obtained in $78 \%$ overall yield from $14 \mathbf{b}$, mp $180 \sim$ $182^{\circ} \mathrm{C}$. NMR $\delta_{\mathrm{H}}\left(\mathrm{CDCl}_{3}\right): 3.12(1 \mathrm{H}, \mathrm{m}), 3.24(1 \mathrm{H}, \mathrm{s}), 3.29$ $(1 \mathrm{H}, \mathrm{dd}, J=4,10 \mathrm{~Hz}), 3.32(3 \mathrm{H}, \mathrm{s}), 3.54(1 \mathrm{H}, \mathrm{dd}, J=2$, $10 \mathrm{~Hz}), 3.72(3 \mathrm{H}, \mathrm{s}), 4.09(1 \mathrm{H}, \mathrm{m}), 4.16(2 \mathrm{H}, \mathrm{s}), 4.34(1 \mathrm{H}$, $\mathrm{dd}, J=6,10 \mathrm{~Hz}), 4.65(1 \mathrm{H}, \mathrm{dd}, J=9,10 \mathrm{~Hz}), 4.93(1 \mathrm{H}, \mathrm{d}$, $J=8 \mathrm{~Hz}), 5.08(1 \mathrm{H}, \mathrm{d}, J=5 \mathrm{~Hz}), 5.97(2 \mathrm{H}, \mathrm{s}), 6.48(1 \mathrm{H}, \mathrm{s})$, $6.84(3 \mathrm{H}, \mathrm{s}), 7.14(1 \mathrm{H}, \mathrm{s})$.

$1-[($ tert-Butyldimethylsilyl)oxy]-6-[6'-methoxy-2'-

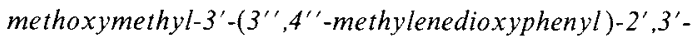
dihydro- $I^{\prime}, 4^{\prime}$-benzodioxin-7'-yl]-3,7-dioxabicyclo[3.3.0]octan-2-ones (17a and 17b). tert-BuMe ${ }_{2} \operatorname{SiOTf}(0.37 \mathrm{ml}$, $1.61 \mathrm{mmol}$ ) dissolved in dry $\mathrm{CH}_{2} \mathrm{Cl}_{2}(2 \mathrm{ml})$ was added in drops to a stirred solution of $15 \mathrm{a}(381 \mathrm{mg}, 0.807 \mathrm{mmol})$ and $2,6-$ lutidine $(0.28 \mathrm{ml}, 2.42 \mathrm{mmol})$ in dry $\mathrm{CH}_{2} \mathrm{Cl}_{2}(6 \mathrm{ml})$ at $-10^{\circ} \mathrm{C}$ under argon. The reaction mixture was gradually warmed up to room temperature and allowed to stand for $17 \mathrm{hr}$. The mixture was then diluted with ether $(30 \mathrm{ml})$ and washed successively with aqueous $\mathrm{Cu}\left(\mathrm{NO}_{3}\right)_{2}$ $(3 \mathrm{~g} / 12 \mathrm{ml} \times 2)$, water and $5 \%$ aqueous $\mathrm{NaHCO}_{3}$. The extract was dried over $\mathrm{Na}_{2} \mathrm{SO}_{4}$ and evaporated. The oily residue was chromatographed on silica gel with $7 \%$ EtOAc in benzene as the eluant to give $17 \mathrm{a}(370 \mathrm{mg}, 0.645 \mathrm{mmol})$ as a colorless viscous oil in $78 \%$ yield. NMR $\delta_{\mathrm{H}}\left(\mathrm{CDCl}_{3}\right)$ : $0.05(3 \mathrm{H}, \mathrm{s}), 0.17(3 \mathrm{H}, \mathrm{s}), 0.80(9 \mathrm{H}, \mathrm{s}), 2.97(1 \mathrm{H}, \mathrm{m}), 3.30$ $(1 \mathrm{H}, \mathrm{dd}, J=6,11 \mathrm{~Hz}), 3.34(3 \mathrm{H}, \mathrm{s}), 3.54(1 \mathrm{H}, \mathrm{dd}, J=3$, $11 \mathrm{~Hz}), 3.72(3 \mathrm{H}, \mathrm{s}), 3.99(1 \mathrm{H}, \mathrm{m}), 4.08(1 \mathrm{H}, \mathrm{d}, J=10 \mathrm{~Hz})$, $4.24(1 \mathrm{H}, \mathrm{d}, J=10 \mathrm{~Hz}), 4.33(1 \mathrm{H}, \mathrm{dd}, J=4,10 \mathrm{~Hz}), 4.58$ $(1 \mathrm{H}, \mathrm{dd}, J=8,9 \mathrm{~Hz}), 4.95(1 \mathrm{H}, \mathrm{d}, J=8 \mathrm{~Hz}), 5.02(1 \mathrm{H}, \mathrm{d}$, $J=5 \mathrm{~Hz}), 5.97(2 \mathrm{H}, \mathrm{s}), 6.47(1 \mathrm{H}, \mathrm{s}), 6.72 \sim 6.88(3 \mathrm{H}, \mathrm{m})$, $7.10(1 \mathrm{H}, \mathrm{s})$.

17b. A viscous oil. NMR $\delta_{\mathrm{H}}\left(\mathrm{CDCl}_{3}\right): 0.07(3 \mathrm{H}, \mathrm{s}), 0.18$ $(3 \mathrm{H}, \mathrm{s}), 0.82(9 \mathrm{H}, \mathrm{s}), 2.93(1 \mathrm{H}, \mathrm{m}), 3.27(1 \mathrm{H}, \mathrm{dd}, J=4$, $11 \mathrm{~Hz}), 3.34(3 \mathrm{H}, \mathrm{s}), 3.58(1 \mathrm{H}, \mathrm{dd}, J=3,11 \mathrm{~Hz}), 3.73(3 \mathrm{H}$, s), $3.97(1 \mathrm{H}, \mathrm{m}), 4.06(1 \mathrm{H}, \mathrm{d}, J=10 \mathrm{~Hz}), 4.25(1 \mathrm{H}, \mathrm{d}, J=$ $10 \mathrm{~Hz}), 4.37(1 \mathrm{H}, \mathrm{dd}, J=4,10 \mathrm{~Hz}), 4.57(1 \mathrm{H}, \mathrm{dd}, J=8$, $9 \mathrm{~Hz}), 4.98(\mathrm{lH}, \mathrm{d}, J=8 \mathrm{~Hz}), 5.05(1 \mathrm{H}, \mathrm{d}, J=5 \mathrm{~Hz}), 5.98$ $(2 \mathrm{H}, \mathrm{s}), 6.48(1 \mathrm{H}, \mathrm{s}), 6.75 \sim 6.90(3 \mathrm{H}, \mathrm{m}), 7.10(1 \mathrm{H}, \mathrm{s})$.

1-[(tert-Butyldimethy/silyl)oxy]-2-hydroxy-6-[6'methoxy-2'-methoxymethyl-3'-(3', $4^{\prime \prime}$-methylenedioxyphenyl)-2',3'-dihydro- $l^{\prime}, 4^{\prime}$-benzodioxin-7'-yl]-3,7-dioxabicyclo[3.3.0]octanes (18a and $\mathbf{1 8 b}$ ). A $1 \mathrm{~m}$ solution of iso- $\mathrm{Bu}_{2} \mathrm{AlH}$ in hexane $(0.815 \mathrm{ml}, 0.815 \mathrm{mmol})$ was added in drops to a stirred solution of $17 \mathrm{a}(308 \mathrm{mg}, 0.526 \mathrm{mmol})$ in dry toluene $(10 \mathrm{ml})$ at $-75^{\circ} \mathrm{C}$ under argon. After $1 \mathrm{hr}$ at $-75^{\circ} \mathrm{C}$, the reaction was quenched by $\mathrm{MeOH}(0.5 \mathrm{ml})$. The mixture was then diluted with ether, washed successively with $0.5 \mathrm{~N} \mathrm{HCl}$ and $5 \%$ aqueous $\mathrm{NaHCO}_{3}$, dried over $\mathrm{Na}_{2} \mathrm{SO}_{4}$, and evaporated. The oily residue was chromatographed on silica gel with $n$-hexane-EtOAc $(2: 1)$ as the eluant to give $18 \mathrm{a}(290 \mathrm{mg}, 0.493 \mathrm{mmol})$ as a viscous oil in $94 \%$ yield. NMR $\delta_{\mathrm{H}}\left(\mathrm{CDCl}_{3}\right): 0.04 \sim 0.28$ $(6 \mathrm{H}, \mathrm{s} \times 4), 0.84 \sim 0.92(9 \mathrm{H}, \mathrm{s} \times 2), 2.28 \sim 2.52(1 \mathrm{H}, \mathrm{m})$, $3.12 \sim 3.36(1 \mathrm{H}, \mathrm{m}), 3.33(3 \mathrm{H}, \mathrm{s}), 3.48 \sim 3.76(1 \mathrm{H}, \mathrm{m}), 3.71$ $(3 \mathrm{H}, \mathrm{s}), 3.80 \sim 4.30(5 \mathrm{H}, \mathrm{m}), 4.65 \sim 5.27(3 \mathrm{H}, \mathrm{m}), 5.98(2 \mathrm{H}$, s), $6.48(1 \mathrm{H}, \mathrm{s}), 6.80 \sim 6.92(3 \mathrm{H}, \mathrm{m}), 7.17(1 \mathrm{H}, \mathrm{s})$.

18b. A viscous oil. NMR $\delta_{\mathrm{H}}\left(\mathrm{CDCl}_{3}\right): 0.05 \sim 0.28(6 \mathrm{H}$, $\mathrm{s} \times 4), 0.76 \sim 0.96(9 \mathrm{H}, \mathrm{s} \times 2), 2.28 \sim 2.68(1 \mathrm{H}, \mathrm{m}), 3.16 \sim$ 3.37 (1H, br.), $3.34(3 \mathrm{H}, \mathrm{s}), 3.46 \sim 3.76(2 \mathrm{H}, \mathrm{m}), 3.71(3 \mathrm{H}$, $\mathrm{s}), 3.84 \sim 4.32(5 \mathrm{H}, \mathrm{m}), 4.75(1 \mathrm{H}, \mathrm{d}, J=8 \mathrm{~Hz}), 4.82 \sim 5.02$ $(1 \mathrm{H}, \mathrm{d} \times 2, J=8 \mathrm{each}), 5.02 \sim 5.25(2 \mathrm{H}, \mathrm{m}), 5.98(2 \mathrm{H}, \mathrm{s})$, $6.47(1 \mathrm{H}, \mathrm{s}), 6.72 \sim 6.92(3 \mathrm{H}, \mathrm{m}), 7.08 \sim 7.20(1 \mathrm{H}, \mathrm{s} \times 2)$.

I-[(tert-Butyldimethylsilyl)oxy]-2-fluoro-6-[6'-methoxy$2^{\prime}$-methoxymethyl-3'-(3', $4^{\prime \prime}$-methylenedioxyphenyl $)-$ $2^{\prime}, 3^{\prime}$-dihydro-1',4'-benzodioxin-7'-yl]-3,7-dioxabicyclo[3.3.0] octane (19a). A solution of $18 \mathbf{a}(290 \mathrm{mg}, 0.493$ mmol), 2-fluoro-1-methylpyridinium tosylate $(0.21 \mathrm{~g}, 0.74$ $\mathrm{mmol})$ and triethylamine $(0.137 \mathrm{ml}, 0.986 \mathrm{mmol})$ in dry $\mathrm{CH}_{2} \mathrm{Cl}_{2}(10 \mathrm{ml})$ was stirred at room temperature for $1.5 \mathrm{hr}$. The solvent was removed under reduced pressure, and the residue was chromatographed on silica gel. Elution with $n$ hexane EtOAc (3:1) afforded 19a (236 $\mathrm{mg}, 0.400 \mathrm{mmol}$ ) as a white powder in $81 \%$ yield. NMR $\delta_{\mathrm{H}}\left(\mathrm{CDCl}_{3}\right): 0.09$ $(6 \mathrm{H}, \mathrm{s}), 0.80 \sim 0.92(9 \mathrm{H}, \mathrm{s} \times 2), 2.53 \sim 2.78(1 \mathrm{H}, \mathrm{m}), 3.10 \sim$ $3.40(1 \mathrm{H}, \mathrm{m}), 3.13(3 \mathrm{H}, \mathrm{s}), 3.40 \sim 3.65(2 \mathrm{H}, \mathrm{m}), 3.70(3 \mathrm{H}$, s), $3.80 \sim 4.48(4 \mathrm{H}, \mathrm{m}), 4.64 \sim 5.04(2 \mathrm{H}, \mathrm{m}), 5.42(0.5 \mathrm{H}, \mathrm{d}$, $J=64 \mathrm{~Hz}), 5.57(0.5 \mathrm{H}, \mathrm{d}, J=64 \mathrm{~Hz}), 5.97(2 \mathrm{H}, \mathrm{s}), 6.46(1 \mathrm{H}$, s), $6.70 \sim 6.96(3 \mathrm{H}, \mathrm{m}), 7.10 \sim 7.20(1 \mathrm{H}, \mathrm{s} \times 2)$.

1-Hydroxy-6-[6'-methoxy-2'-methoxymethyl-3'-(3', $4^{\prime \prime}$ methylenedioxyphenyl)-2', 3'-dihydro- $1^{\prime}, 4^{\prime}$-benzodioxin-7'$y l]-2-\left(2^{\prime \prime \prime}\right.$-methoxy-3" $3^{\prime \prime}, 4^{\prime \prime \prime}$-methylenedioxyphenoxy)-3,7dioxabicyclo[3.3.0]octanes (2a, 2b, 28a and $\mathbf{2 8 b}$ ).

Method A. Trimethylsilyl trifluoromethanesulfonate $(7.5 \mu \mathrm{l}, 0.004 \mathrm{mmol})$ was injected via a syringe into a solution of fluoride $19 \mathrm{a}(50 \mathrm{mg}, 0.085 \mathrm{mmol})$ and silyl ether $21(29 \mathrm{mg}, 0.121 \mathrm{mmol})$ in dry ether $(2 \mathrm{ml})$ at $0^{\circ} \mathrm{C}$. After $4 \mathrm{hr}$ at $0^{\circ} \mathrm{C}$, one drop of triethylamine was added, and the mixture was concentrated under reduced pressure. The crude product was purified by preparative TLC on silica gel developed with 10\% EtOAc in benzene to give 25a $(50 \mathrm{mg}, 0.068 \mathrm{mmol})$ in $80 \%$ yield.

A $2 \alpha / 2 \beta$ mixture of silyl ethers $25 a$ was dissolved in dry THF $(1 \mathrm{ml})$, and a $1 \mathrm{M}$ solution of $n-\mathrm{Bu}_{4} \mathrm{NF}$ in THF $(0.10 \mathrm{ml}, 0.10 \mathrm{mmol})$ was added. After $1.5 \mathrm{hr}$ at room temperature, the mixture was diluted with $\mathrm{CH}_{2} \mathrm{Cl}_{2}$, washed with $10 \%$ aqueous $\mathrm{NH}_{4} \mathrm{Cl}$, dried over $\mathrm{Na}_{2} \mathrm{SO}_{4}$, and concentrated. The residue was purified by preparative TLC on silica gel developed with $20 \%$ EtOAc in benzene to give $2 \mathrm{a}(7.8 \mathrm{mg}, 0.013 \mathrm{mmol})$ along with $28 \mathrm{a}(30.7 \mathrm{mg}$, $0.049 \mathrm{mmol}$ ) in $19 \%$ and $72 \%$ yields, respectively).

Method B. A mixture of fluoride 19a (39 $\mathrm{mg}, 0.066$ $\mathrm{mmol})$, 2-methoxy-4,5-methylenedioxyphenol $(22,13 \mathrm{mg}$, $0.079 \mathrm{mmol}), \mathrm{TrClO}_{4}(27 \mathrm{mg}, 0.079 \mathrm{mmol}), \mathrm{SnCl}_{2}(26 \mathrm{mg}$, $0.133 \mathrm{mmol}$ ) and pulverized $4 \mathrm{~A}$ molecular sieves in dry ether $(3 \mathrm{ml})$ was stirred at $0 \sim 5^{\circ} \mathrm{C}$ for $5.5 \mathrm{hr}$. The reaction was then quenched by adding $5 \%$ aqueous $\mathrm{NaHCO}_{3}$, and the resulting mixture was filtered through a pad of celite with the aid of ether. The ethereal layer was separated, washed with $0.5 \mathrm{~N}$ aqueous $\mathrm{NaOH}$, dried over $\mathrm{Na}_{2} \mathrm{SO}_{4}$, and concentrated. The oily residue was purified by pre- 
parative TLC on silica gel developed with $10 \%$ EtOAc in benzene to give 25 a ( $39 \mathrm{mg}, 0.053 \mathrm{mmol}$ ) in $80 \%$ yield.

Silyl ethers 25a were desilylated with $n$ - $\mathrm{Bu}_{4} \mathrm{NF}$ in THF to give $\mathbf{2 a}$ and $\mathbf{2 8 a}$ in a ratio of $31: 69$ (determined by HPLC).

2a $\left[\left( \pm\right.\right.$ )-haedoxan D]. mp $155 \sim 157^{\circ} \mathrm{C}$ (ether). NMR $\delta_{\mathrm{H}}$ $\left(\mathrm{CDCl}_{3}\right): 1.62(1 \mathrm{H}, \mathrm{br}), 2.64(1 \mathrm{H}, \mathrm{m}), 3.28(1 \mathrm{H}, \mathrm{dd}, J=5$, $11 \mathrm{~Hz}), 3.34(3 \mathrm{H}, \mathrm{s}), 3.58(1 \mathrm{H}, \mathrm{dd}, J=3,11 \mathrm{~Hz}), 3.72(3 \mathrm{H}$, s), $3.74(1 \mathrm{H}, \mathrm{d}, J=11 \mathrm{~Hz}), 3.77(3 \mathrm{H}, \mathrm{s}), 3.87 \sim 4.15(2 \mathrm{H}$, $\mathrm{m}), 4.29(1 \mathrm{H}, \mathrm{d}, J=11 \mathrm{~Hz}), 4.44(1 \mathrm{H}, \mathrm{dd}, J=8,10 \mathrm{~Hz})$, $4.92(1 \mathrm{H}, \mathrm{d}, J=6 \mathrm{~Hz}), 4.96(1 \mathrm{H}, \mathrm{d}, J=8 \mathrm{~Hz}), 5.17(1 \mathrm{H}, \mathrm{s})$, $5.88(2 \mathrm{H}, \mathrm{s}), 5.98(2 \mathrm{H}, \mathrm{s}), 6.47(1 \mathrm{H}, \mathrm{s}), 6.53(1 \mathrm{H}, \mathrm{s}), 6.76$ $(1 \mathrm{H}, \mathrm{s}), 6.80 \sim 6.98(3 \mathrm{H}, \mathrm{m}), 7.28(1 \mathrm{H}, \mathrm{s})$.

28a. mp $172 \sim 173^{\circ} \mathrm{C}$. NMR $\delta_{\mathrm{H}}\left(\mathrm{CDCl}_{3}\right): 2.62(1 \mathrm{H}$, br. $)$, $2.72(1 \mathrm{H}, \mathrm{q}, J=7 \mathrm{~Hz}), 3.27(1 \mathrm{H}, \mathrm{dd}, J=4,11 \mathrm{~Hz}), 3.33$ $(3 \mathrm{H}, \mathrm{s}), 3.58(1 \mathrm{H}, \mathrm{dd}, J=3,11 \mathrm{~Hz}), 3.71(3 \mathrm{H}, \mathrm{s}), 3.79(3 \mathrm{H}$, s), $3.98(1 \mathrm{H}, \mathrm{m}), 4.05(1 \mathrm{H}, \mathrm{d}, J=10 \mathrm{~Hz}), 4.20(2 \mathrm{H}, \mathrm{d}, J=$ $7 \mathrm{~Hz}), 4.57(1 \mathrm{H}, \mathrm{d}, J=10 \mathrm{~Hz}), 4.97(1 \mathrm{H}, \mathrm{d}, J=8 \mathrm{~Hz}), 5.02$ $(1 \mathrm{H}, \mathrm{d}, J=7 \mathrm{~Hz}), 5.34(1 \mathrm{H}, \mathrm{s}), 5.87(2 \mathrm{H}, \mathrm{s}), 5.97(2 \mathrm{H}, \mathrm{s})$, $6.47(1 \mathrm{H}, \mathrm{s}), 6.54(1 \mathrm{H}, \mathrm{s}), 6.74(1 \mathrm{H}, \mathrm{s}), 6.80 \sim 6.90(3 \mathrm{H}, \mathrm{m})$, $7.18(1 \mathrm{H}, \mathrm{s})$. Anal. Found: C, 61.13; H, 5.18. Calcd. for $\mathrm{C}_{32} \mathrm{H}_{32} \mathrm{O}_{13}: \mathrm{C}, 61.54 ; \mathrm{H}, 5.16 \%$.

Compounds $\mathbf{2 b}$ and $\mathbf{2 8 b}$, isomers of the benzodioxane part of $2 \mathbf{a}$ and $\mathbf{2 8 a}$, were obtained from $19 \mathbf{b}$ by "method A" for synthesizing $\mathbf{2 a}$ and $\mathbf{2 8 a}$.

2b. $\operatorname{mp} 194 \sim 196^{\circ} \mathrm{C}$. NMR $\delta_{\mathrm{H}}\left(\mathrm{CDCl}_{3}\right): 1.70(1 \mathrm{H}$, br. $)$, $2.63(1 \mathrm{H}, \mathrm{m}), 3.28(1 \mathrm{H}, \mathrm{dd}, J=4,11 \mathrm{~Hz}), 3.33(3 \mathrm{H}, \mathrm{s}), 3.54$ $(1 \mathrm{H}, \mathrm{dd}, J=3,11 \mathrm{~Hz}), 3.71(3 \mathrm{H}, \mathrm{s}), 3.73(1 \mathrm{H}, \mathrm{d}, J=10 \mathrm{~Hz})$, $3.75(3 \mathrm{H}, \mathrm{s}), 3.84 \sim 4.15(3 \mathrm{H}, \mathrm{m}), 4.29(1 \mathrm{H}, \mathrm{d}, J=10 \mathrm{~Hz})$, $4.43(1 \mathrm{H}, \mathrm{dd}, J=7,10 \mathrm{~Hz}), 4.88(1 \mathrm{H}, \mathrm{d}, J=6 \mathrm{~Hz}), 4.94$ $(1 \mathrm{H}, \mathrm{d}, J=8 \mathrm{~Hz}), 5.16(1 \mathrm{H}, \mathrm{s}), 5.87(2 \mathrm{H}, \mathrm{s}), 5.96(2 \mathrm{H}, \mathrm{s})$, $6.45(1 \mathrm{H}, \mathrm{s}), 6.52(1 \mathrm{H}, \mathrm{s}), 6.76(1 \mathrm{H}, \mathrm{s}), 6.78 \sim 6.95(3 \mathrm{H}, \mathrm{m})$, $7.26(1 \mathrm{H}, \mathrm{s})$.

28b. mp $147 \sim 149^{\circ} \mathrm{C}$. NMR $\delta_{\mathrm{H}}\left(\mathrm{CHCl}_{3}\right): 2.72(1 \mathrm{H}, \mathrm{m})$, $2.79(1 \mathrm{H}$, br. $), 3.29(1 \mathrm{H}, \mathrm{dd}, J=4,11 \mathrm{~Hz}), 3.33(3 \mathrm{H}, \mathrm{s}), 3.54$ $(1 \mathrm{H}, \mathrm{dd}, J=3,11 \mathrm{~Hz}), 3.71(3 \mathrm{H}, \mathrm{s}), 3.78(3 \mathrm{H}, \mathrm{s}), 3.96(1 \mathrm{H}$, $\mathrm{m}), 4.04(\mathrm{IH}, \mathrm{d}, J=10 \mathrm{~Hz}), 4.20(2 \mathrm{H}, \mathrm{d}, J=7 \mathrm{~Hz}), 4.55$ $(1 \mathrm{H}, \mathrm{d}, J=10 \mathrm{~Hz}), 4.93(1 \mathrm{H}, \mathrm{d}, J=8 \mathrm{~Hz}), 5.00(1 \mathrm{H}, \mathrm{d}, J=$ $6 \mathrm{~Hz}), 5.33(1 \mathrm{H}, \mathrm{s}), 5.87(2 \mathrm{H}, \mathrm{s}), 5.97(2 \mathrm{H}, \mathrm{s}), 6.46(1 \mathrm{H}, \mathrm{s})$, $6.54(1 \mathrm{H}, \mathrm{s}), 6.73(1 \mathrm{H}, \mathrm{s}), 6.77 \sim 6.94(3 \mathrm{H}, \mathrm{m}), 7.18(1 \mathrm{H}, \mathrm{s})$.

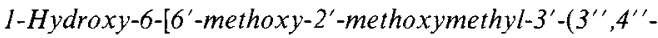
methylenedioxyphenyl)-2',3'-dihydro-I',4'-benzodioxin-7'yl]-2-(3"', $4^{\prime \prime \prime}$-methylenedioxyphenoxy)-3,7-dioxabicyclo[3.3.0]octanes (3a, 3b, 29a and 29b). Compounds 3a and 29a were obtained via 26a by "method $\mathrm{A}$ " from fluoride 19a and $O$-trimethylsilylsesamol (20) in $25 \%$ and $61 \%$ yields, respectively.

3a $\left[( \pm)\right.$-haedoxan E]. mp $158 \sim 159^{\circ} \mathrm{C}$ (ether). NMR $\delta_{\mathrm{H}}$ $\left(\mathrm{CDCl}_{3}\right): 2.58(1 \mathrm{H}, \mathrm{m}), 3.10(1 \mathrm{H}, \mathrm{d}, J=1 \mathrm{~Hz}), 3.27(1 \mathrm{H}$, $\mathrm{dd}, J=4,11 \mathrm{~Hz}), 3.35(3 \mathrm{H}, \mathrm{s}), 3.58(1 \mathrm{H}, \mathrm{dd}, J=3,11 \mathrm{~Hz})$, $3.72(3 \mathrm{H}, s), 3.77(1 \mathrm{H}, \mathrm{dd}, J=1,10 \mathrm{~Hz}), 3.88 \sim 4.13(2 \mathrm{H}$, $\mathrm{m}), 4.32(1 \mathrm{H}, \mathrm{d}, J=10 \mathrm{~Hz}), 4.36(1 \mathrm{H}, \mathrm{dd}, J=6,10 \mathrm{~Hz})$, $4.92(1 \mathrm{H}, \mathrm{d}, J=6 \mathrm{~Hz}), 4.98(1 \mathrm{H}, \mathrm{d}, J=8 \mathrm{~Hz}), 5.27(1 \mathrm{H}, \mathrm{s})$, $5.92(2 \mathrm{H}, \mathrm{s}), 5.97(2 \mathrm{H}, \mathrm{s}), 6.47(1 \mathrm{H}, \mathrm{s}), 6.48 \sim 6.96(6 \mathrm{H}, \mathrm{m})$, $7.27(1 \mathrm{H}, \mathrm{s})$. NMR $\delta_{\mathrm{C}}\left(\mathrm{CDCl}_{3}\right): 55.7(\mathrm{q}), 58.2(\mathrm{~d}), 59.5(\mathrm{q})$, $70.9(\mathrm{t}), 71.2(\mathrm{t}), 76.5(\mathrm{~d}), 77.2(\mathrm{t}), 83.9(\mathrm{~d}), 92.1(\mathrm{~s}), 99.9$ (d), $100.5(\mathrm{~d}), 101.2(\mathrm{t} \times 2), 103.4(\mathrm{~d}), 107.6(\mathrm{~d}), 108.0(\mathrm{~d})$, $108.4(\mathrm{~d}), 109.8$ (d), 115.1 (d), 121.2 (d), 122.9 (s), $130.5(\mathrm{~s})$, $136.9(\mathrm{~s}), 143.0(\mathrm{~s}), 143.3(\mathrm{~s}), 148.0(\mathrm{~s} \times 3), 150.5(\mathrm{~s}), 151.3$ (s). Anal. Found: C, 62.74; $\mathrm{H}, 5.31$. Calcd. for $\mathrm{C}_{31} \mathrm{H}_{30} \mathrm{O}_{12}$ : C, 62.62; H, 5.09\%.

29a. $\mathrm{mp} 169 \sim 171^{\circ} \mathrm{C}$. NMR $\delta_{\mathrm{H}}\left(\mathrm{CDCl}_{3}\right): 2.48(1 \mathrm{H}$, br. $)$, $2.72(1 \mathrm{H}, \mathrm{m}), 3.27(1 \mathrm{H}, \mathrm{dd}, J=4,11 \mathrm{~Hz}), 3.33(3 \mathrm{H}, \mathrm{s}), 3.58$ $(1 \mathrm{H}, \mathrm{dd}, J=3,11 \mathrm{~Hz}), 3.72(3 \mathrm{H}, \mathrm{s}), 3.87 \sim 4.31(3 \mathrm{H}, \mathrm{m})$, $4.26(1 \mathrm{H}, \mathrm{dd}, J=8,9 \mathrm{~Hz}), 4.45(1 \mathrm{H}, \mathrm{d}, J=11 \mathrm{~Hz}), 4.95$ $(1 \mathrm{H}, \mathrm{d}, J=6 \mathrm{~Hz}), 4.97(1 \mathrm{H}, \mathrm{d}, J=8 \mathrm{~Hz}), 5.41(1 \mathrm{H}, \mathrm{s}), 5.90$ $(2 \mathrm{H}, \mathrm{s}), 5.97(2 \mathrm{H}, \mathrm{s}), 6.48(1 \mathrm{H}, \mathrm{s}), 6.52 \sim 6.97(6 \mathrm{H}, \mathrm{m}), 7.16$ $(1 \mathrm{H}, \mathrm{s})$.

Compounds $\mathbf{3 b}$ and $\mathbf{2 9}$, isomers of the benzodioxane part of $3 \mathbf{a}$ and $29 \mathbf{a}$, were obtained from $19 \mathbf{b}$ by "method A" for producing $2 \mathbf{a}$ and $\mathbf{2 8 a}$.

3b. Resinous. NMR $\delta_{\mathrm{H}}\left(\mathrm{CDCl}_{3}\right): 2.62(1 \mathrm{H}, \mathrm{m}), 3.11(1 \mathrm{H}$, $\mathrm{d}, J=1 \mathrm{~Hz}), 3.29(1 \mathrm{H}, \mathrm{dd}, J=5,11 \mathrm{~Hz}), 3.34(3 \mathrm{H}, \mathrm{s}), 3.55$ $(1 \mathrm{H}, \mathrm{dd}, J=3,11 \mathrm{~Hz}), 3.72(3 \mathrm{H}, \mathrm{s}), 3.77(1 \mathrm{H}, \mathrm{dd}, J=1$, $9 \mathrm{~Hz}), 3.90 \sim 4.19(2 \mathrm{H}, \mathrm{m}), 4.32(1 \mathrm{H}, \mathrm{d}, J=9 \mathrm{~Hz}), 4.35(1 \mathrm{H}$, dd, $J=7,9 \mathrm{~Hz}), 4.88(1 \mathrm{H}, \mathrm{d}, J=6 \mathrm{~Hz}), 4.95(1 \mathrm{H}, \mathrm{d}, J=$ $8 \mathrm{~Hz}), 5.28(1 \mathrm{H}, \mathrm{s}), 5.92(2 \mathrm{H}, \mathrm{s}), 5.98(2 \mathrm{H}, \mathrm{s}), 6.47(1 \mathrm{H}, \mathrm{s})$, $6.52 \sim 6.96(6 \mathrm{H}, \mathrm{m}), 7.27(1 \mathrm{H}, \mathrm{s})$.

29b. mp $156 \sim 158^{\circ} \mathrm{C} . \mathrm{NMR} \delta_{\mathrm{H}}\left(\mathrm{CDCl}_{3}\right): 2.45(1 \mathrm{H}, \mathrm{br}$.$) ,$ $2.75(1 \mathrm{H}, \mathrm{m}), 3.29(1 \mathrm{H}, \mathrm{dd}, J=5,11 \mathrm{~Hz}), 3.33(3 \mathrm{H}, \mathrm{s}), 3.55$ $(1 \mathrm{H}, \mathrm{dd}, J=3,11 \mathrm{~Hz}), 3.72(3 \mathrm{H}, \mathrm{s}), 3.90 \sim 4.16(3 \mathrm{H}, \mathrm{m})$, $4.27(1 \mathrm{H}, \mathrm{dd}, J=8,9 \mathrm{~Hz}), 4.45(1 \mathrm{H}, \mathrm{d}, J=10 \mathrm{~Hz}), 4.93$ $(1 \mathrm{H}, \mathrm{d}, J=6 \mathrm{~Hz}), 4.95(1 \mathrm{H}, \mathrm{d}, J=8 \mathrm{~Hz}), 5.42(1 \mathrm{H}, \mathrm{s}), 5.91$ $(2 \mathrm{H}, \mathrm{s}), 5.98(2 \mathrm{H}, \mathrm{s}), 6.48(1 \mathrm{H}, \mathrm{s}), 6.52 \sim 6.92(6 \mathrm{H}, \mathrm{m}), 7.16$ $(1 \mathrm{H}, \mathrm{s})$.

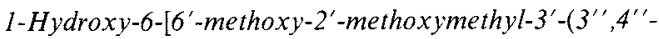
methylenedioxyphenyl)-2',3'-dihydro- $I^{\prime}, 4^{\prime}$-benzodioxin- $7^{\prime}$ $y l]-2-\left(2 " \prime, 6 "\right.$ "'-dime thoxy-3" ${ }^{\prime \prime}, 4 "$ "-methylenedioxyphenoxy)-3,7-dioxabicyclo[3.3.0]octanes (1a, 1b, 27a and 27b). Compounds 1a and 27a were obtained in $17 \%$ and $16 \%$ yields, respectively, by the reaction between fluoride 19a and 2,6-dimethoxy-3,4-methylenedioxyphenol (23) by a similar procedure to that of "method B", using $\mathrm{AgClO}_{4}$ instead of $\mathrm{TrClO}_{4}$

1a $\left[( \pm)\right.$-haedoxan A]. mp $173 \sim 175^{\circ} \mathrm{C}$. NMR $\delta_{\mathrm{H}}$ $\left(\mathrm{CDCl}_{3}\right): 1.64(1 \mathrm{H}$, br. $), 2.63(1 \mathrm{H}, \mathrm{m}), 3.27(1 \mathrm{H}, \mathrm{dd}, J=4$, $11 \mathrm{~Hz}), 3.34(3 \mathrm{H}, \mathrm{s}), 3.58(1 \mathrm{H}, \mathrm{dd}, J=3,11 \mathrm{~Hz}), 3.71(1 \mathrm{H}$, d, $J=10 \mathrm{~Hz}), 3.72(3 \mathrm{H}, \mathrm{s}), 3.77(3 \mathrm{H}, \mathrm{s}), 3.88 \sim 4.13(3 \mathrm{H}$, $\mathrm{m}), 4.00(3 \mathrm{H}, \mathrm{s}), 4.28(1 \mathrm{H}, \mathrm{d}, J=10 \mathrm{~Hz}), 4.55(1 \mathrm{H}, \mathrm{dd}, J=$ $7,9 \mathrm{~Hz}), 4.91(1 \mathrm{H}, \mathrm{d}, J=5 \mathrm{~Hz}), 4.98(1 \mathrm{H}, \mathrm{d}, J=8 \mathrm{~Hz}), 5.22$ $(1 \mathrm{H}, \mathrm{s}), 5.85(2 \mathrm{H}, \mathrm{s}), 5.97(2 \mathrm{H}, \mathrm{s}), 6.27(1 \mathrm{H}, \mathrm{s}), 6.47(1 \mathrm{H}, \mathrm{s})$, $6.79 \sim 6.96(3 \mathrm{H}, \mathrm{m}), 7.28(1 \mathrm{H}, \mathrm{s})$. Anal. Found: C, 60.65 ; $\mathrm{H}, 5.69$. Caled. for $\mathrm{C}_{33} \mathrm{H}_{34} \mathrm{O}_{14}: \mathrm{C}, 60.55 ; \mathrm{H}, 5.24 \%$.

27a: mp $196 \sim 198^{\circ} \mathrm{C}$. NMR $\delta_{\mathrm{H}}\left(\mathrm{CDCl}_{3}\right): 2.70(2 \mathrm{H}, \mathrm{m})$, $3.27(1 \mathrm{H}, \mathrm{dd}, J=4,11 \mathrm{~Hz}), 3.33(3 \mathrm{H}, \mathrm{s}), 3.57(1 \mathrm{H}, \mathrm{dd}, J=$ $3,11 \mathrm{~Hz}), 3.72(3 \mathrm{H}, \mathrm{s}), 3.78(3 \mathrm{H}, \mathrm{s}), 3.99(1 \mathrm{H}, \mathrm{m}), 4.00(3 \mathrm{H}$, s), $4.07(1 \mathrm{H}, \mathrm{d}, J=10 \mathrm{~Hz}), 4.16(1 \mathrm{H}, \mathrm{dd}, J=8,9 \mathrm{~Hz}), 4.33$ $(1 \mathrm{H}, \mathrm{dd}, J=6,9 \mathrm{~Hz}), 4.61(1 \mathrm{H}, \mathrm{d}, J=10 \mathrm{~Hz}), 4.95(1 \mathrm{H}, \mathrm{d}$, $J=8 \mathrm{~Hz}), 5.09(1 \mathrm{H}, \mathrm{d}, J=7 \mathrm{~Hz}), 5.35(1 \mathrm{H}, \mathrm{s}), 5.84(2 \mathrm{H}, \mathrm{q}$, $J=1 \mathrm{~Hz}), 5.97(2 \mathrm{H}, \mathrm{s}), 6.27(1 \mathrm{H}, \mathrm{s}), 6.46(1 \mathrm{H}, \mathrm{s}), 6.72 \sim$ $6.96(3 \mathrm{H}, \mathrm{m}), 7.21(1 \mathrm{H}, \mathrm{s})$.

$2 \alpha, 6 \beta$-isomer. Resinous. NMR $\delta_{\mathrm{H}}\left(\mathrm{CDCl}_{3}\right): 3.07(1 \mathrm{H}, \mathrm{m})$, 
m), $3.28(1 \mathrm{H}, \mathrm{dd}, J=4,11 \mathrm{~Hz}), 3.34(3 \mathrm{H}, \mathrm{s}), 3.44 \sim 3.68$ $(2 \mathrm{H}, \mathrm{m}), 3.71(3 \mathrm{H}, \mathrm{s}), 3.79(3 \mathrm{H}, \mathrm{s}), 3.83 \sim 4.16(4 \mathrm{H}, \mathrm{m})$, $4.98(1 \mathrm{H}, \mathrm{d}, J=8 \mathrm{~Hz}), 5.17(1 \mathrm{H}, \mathrm{s}), 5.31(1 \mathrm{H}, \mathrm{d}, J=5 \mathrm{~Hz})$, $5.86(2 \mathrm{H}, \mathrm{s}), 5.98(2 \mathrm{H}, \mathrm{s}), 6.44(1 \mathrm{H}, \mathrm{s}), 6.52(1 \mathrm{H}, \mathrm{s}), 6.73$ $(1 \mathrm{H}, \mathrm{s}), 6.76 \sim 6.93(3 \mathrm{H}, \mathrm{m}), 7.17(1 \mathrm{H}, \mathrm{s})$.

Compounds $\mathbf{1 b}$ and $\mathbf{2 7 b}$, isomers of the benzodioxane part of $1 \mathrm{a}$ and $27 \mathrm{a}$, were obtained from $19 \mathrm{~b}$ by "method A" for producing $\mathbf{2 a}$ and $\mathbf{2 8 a}$.

1b. $\mathrm{mp} 139 \sim 140^{\circ} \mathrm{C}$. NMR $\delta_{\mathrm{H}}\left(\mathrm{CDCl}_{3}\right): 2.63(\mathrm{lH}, \mathrm{m})$, $3.29(1 \mathrm{H}, \mathrm{dd}, J=4,11 \mathrm{~Hz}), 3.33(3 \mathrm{H}, \mathrm{s}), 3.54(1 \mathrm{H}, \mathrm{dd}, J=3$, $11 \mathrm{~Hz}), 3.71(3 \mathrm{H}, \mathrm{s}), 3.72(1 \mathrm{H}, \mathrm{d}, J=10 \mathrm{~Hz}), 3.76(3 \mathrm{H}, \mathrm{s})$, $3.84 \sim 4.12(3 \mathrm{H}, \mathrm{m}), 4.00(3 \mathrm{H}, \mathrm{s}), 4.28(1 \mathrm{H}, \mathrm{d}, J=10 \mathrm{~Hz})$, $4.56(1 \mathrm{H}, \mathrm{dd}, J=7,9 \mathrm{~Hz}), 4.87(1 \mathrm{H}, \mathrm{d}, J=6 \mathrm{~Hz}), 4.94(1 \mathrm{H}$, $\mathrm{d}, J=8 \mathrm{~Hz}), 5.20(1 \mathrm{H}, \mathrm{s}), 5.84(2 \mathrm{H}, \mathrm{s}), 5.96(2 \mathrm{H}, \mathrm{s}), 6.26$ $(1 \mathrm{H}, \mathrm{s}), 6.46(1 \mathrm{H}, \mathrm{s}), 6.72 \sim 6.95(3 \mathrm{H}, \mathrm{m}), 7.27(1 \mathrm{H}, \mathrm{s})$.

27b. $\mathrm{mp} 157 \sim 158^{\circ} \mathrm{C}$. NMR $\delta_{\mathrm{H}}\left(\mathrm{CDCl}_{3}\right): 2.52 \sim 2.84$ $(2 \mathrm{H}, \mathrm{m}), 3.28(1 \mathrm{H}, \mathrm{dd}, J=4,11 \mathrm{~Hz}), 3.33(3 \mathrm{H}, \mathrm{s}), 3.53(1 \mathrm{H}$, $\mathrm{dd}, J=3,11 \mathrm{~Hz}), 3.71(3 \mathrm{H}, \mathrm{s}), 3.77(3 \mathrm{H}, \mathrm{s}), 3.98(1 \mathrm{H}, \mathrm{m})$, $4.00(3 \mathrm{H}, \mathrm{s}), 4.03(1 \mathrm{H}, \mathrm{d}, J=10 \mathrm{~Hz}), 4.15(1 \mathrm{H}, \mathrm{dd}, J=8$, $9 \mathrm{~Hz}), 4.34(1 \mathrm{H}, \mathrm{dd}, J=6,9 \mathrm{~Hz}), 4.60(1 \mathrm{H}, \mathrm{d}, J=10 \mathrm{~Hz})$, $4.93(1 \mathrm{H}, \mathrm{d}, J=8 \mathrm{~Hz}), 5.07(1 \mathrm{H}, \mathrm{d}, J=7 \mathrm{~Hz}), 5.35(1 \mathrm{H}, \mathrm{s})$, $5.84(2 \mathrm{H}, \mathrm{s}), 5.96(2 \mathrm{H}, \mathrm{s}), 6.27(1 \mathrm{H}, \mathrm{s}), 6.45(1 \mathrm{H}, \mathrm{s})$, $6.68 \sim 6.94(3 \mathrm{H}, \mathrm{m}), 7.20(1 \mathrm{H}, \mathrm{s})$.

\section{References}

1) F. Ishibashi and E. Taniguchi, Agric. Biol. Chem., 50, 3119 (1986).

2) F. Ishibashi and E. Taniguchi, Chem. Lett., 1986,
1771.

3) F. Ishibashi and E. Taniguchi, Bull. Chem. Soc. Jpn., 61, 4361 (1988).

4) E. Taniguchi and F. Ishibashi, Chem. Lett., 1989, 313.

5) E. Taniguchi, K. Imamura, F. Ishibashi, T. Matsui and A. Nishio, Agric. Biol. Chem, 53, 631 (1989).

6) E. Taniguchi, in preparation.

7) F. Ishibashi and E. Taniguchi, Agric. Biol. Chem., 53, 1557 (1989).

8) K. Kondo and F. Mori, Chem. Lett., 1974, 741.

9) E. J. Corey, H. Cho, C. Rüker and D. H. Hua, Tetrahedron Lett., 22, 3455 (1981).

10) H. O. House, D. S. Crumrine, A. Y. Teranishi and H. Olmstead, J. Am. Chem. Soc., 95, 3310 (1973).

11) V. VanRheenen, R. C. Kelly and D. Y. Cha, Tetrahedron Lett., 1976, 1973.

12) V. Balogh, M. Fetizon and M. Golfie, J. Org. Chem., 36, 1339 (1971)

13) E. J. Corey and A. Venkateswarlu, J. Am. Chem. Soc., 94, 6190 (1972).

14) The signs of $R^{*} / S^{*}$ were tentatively used to indicate the difference between the configurations of $15 \mathbf{a}$ and 15b.

15) T. Mukaiyama, Y. Hashimoto and S. Shoda, Chem. Lett., 1983, 935.

16) S. Hashimoto, M. Hayashi and R. Noyori, Tetrahedron Lett., 25, 1379 (1984).

17) T. Mukaiyama, Y. Murai and S. Shoda, Chem. Lett., 1981, 43 I. 